\title{
Aggregated mobility data could help fight COVID-19
}

\author{
Caroline O. Buckee ${ }^{1 *}$, Satchit Balsari² ${ }^{2}$ Jennifer Chan $^{3}$, Mercè Crosas $^{4}$, Francesca Dominici $^{5}$, Urs Gasser $^{6}$, \\ Yonatan H. Grad ${ }^{1}$, Bryan Grenfell ${ }^{7}$, M. Elizabeth Halloran ${ }^{8,9}$, Moritz U. G. Kraemer ${ }^{10,11}$, Marc Lipsitch ${ }^{1}$, C. \\ Jessica E. Metcalf ${ }^{7}$, Lauren Ancel Meyers ${ }^{12}$, T. Alex Perkins ${ }^{13}$, Mauricio Santillana ${ }^{14,11}$, Samuel V. Scarpino ${ }^{15}$, \\ Cecile Viboud $^{16}$, Amy Wesolowski ${ }^{17}$, Andrew Schroeder ${ }^{18}$
}

\begin{abstract}
${ }^{1}$ Center for Communicable Disease Dynamics, Harvard T.H. Chan School of Public Health, Boston, MA 02115 USA. ${ }^{2}$ Emergency Medicine, Beth Israel Deaconess Medical Center, Harvard Medical School, Boston, MA 02115, USA. ${ }^{3}$ Emergency Medicine, Feinberg School of Medicine, Northwestern University, IL 60611. ${ }^{4}$ Institute for Quantitative Social Science, Harvard University, Boston, MA 02138, USA. ${ }^{5 H a r v a r d ~ D a t a ~ S c i e n c e ~ I n i t i a t i v e, ~ H a r v a r d ~ U n i v e r s i t y, ~ B o s t o n, ~ M A ~ 02138, ~ U S A . ~}{ }^{6}$ Berkman Klein Center for Internet and Society, Harvard University Harvard Law School, Boston, MA 02138, USA. ${ }^{7 P}$ rinceton University, Princeton, NJ 08540, USA. ${ }^{8} \mathrm{Center}$ for Inference and Dynamics of Infectious Diseases, Fred Hutchinson Cancer Research Center, Seattle, WA 98109, USA. ${ }^{9}$ University of Washington, Seattle, WA 98195, USA. ${ }^{10}$ Department of Zoology, University of Oxford, Oxford OX1 3SZ, UK. "11Boston Children's Hospital, Boston, MA 02115, USA. ${ }^{12}$ The University of Texas at Austin, Austin, TX 78702, USA. ${ }^{13}$ Department of Biological Sciences and Eck Institute for Global Health, University of Notre Dame, Notre Dame, IN 46556, USA. ${ }^{14}$ Department of Pediatrics, Harvard Medical School, Boston, MA 02115, USA. ${ }^{15}$ Network Science Institute, Northeastern University, Boston, MA 02115, USA. ${ }^{16}$ Fogarty International Center, National Institutes of Health, Bethesda, MA 20892, USA. ${ }^{17}$ Department of Epidemiology, Johns Hopkins Bloomberg School of Public Health, Baltimore, MD 21205, USA. 18Direct Relief, Santa Barbara, CA 93117, USA. ${ }^{*}$ Corresponding author. Email: cbuckee@hsph.harvard.edu
\end{abstract}

As the coronavirus disease (COVID-19) epidemic worsens, understanding the effectiveness of public messaging and large-scale social distancing interventions is critical. The research and public health response communities can and should use population mobility data collected by private companies, with appropriate legal, organizational, and computational safeguards in place. When aggregated, these data can help refine interventions by providing near realtime information about changes in patterns of human movement.

Research groups and nonprofit humanitarian agencies have refined data use agreements to stipulate clear guidelines that ensure responsible data practices (1). New tools for specifying different levels of privacy for different users and providing privacy-preserving results, such as the OpenDP platform (2), will effectively manage data access, and aggregation steps have been carefully reviewed on a legal and methodological basis to ensure that the analyses follow ethical guidelines for human participants (3). To monitor social distancing interventions, for example, rather than showing individual travel or behavior patterns, information from multiple devices is aggregated in space and time, so that the data reflects an approximation of population-level mobility (4).

The estimates of aggregate flows of people are incredibly valuable. A map that examines the impact of social distancing messaging or policies on population mobility patterns, for example, will help county officials understand what kinds of messaging or policies are most effective. Comparing the public response to interventions, in terms of the rate of movement over an entire county from one day to the next, measured against a baseline from normal times, can provide insight into the degree to which recommendations on social distancing are being followed. We will need these estimates, not only now but also when we need to resume life again without risking a major resurgence.

The protection of personal privacy must be paramount.
Consent-based data sharing models and data protection laws provide for the legal grounds to use personal data during emergencies, but we do not advocate the use of individual data $(5,6)$. The measures proposed do not need to run afoul of data protection goals, as a recent statement by the Chair of the European Data Protection Board in the context of the COVID-19 outbreak clarifies (7).

There are already precedents in Asia and Europe (8). Deutsche Telekom has shared aggregated data with Germany to help measure social distancing, in compliance with EU laws (9). The more such analyses are initiated and concluded openly, and in accordance with the law, the greater will be the public trust and our ability to produce reliable analytic insights. Associated risks should be thoughtfully addressed and weighed against the benefits of the data, which could help reduce the death toll in vulnerable populations.

\section{REFERENCES AND NOTES}

1. F. Greenwood et al., "The signal code: A human rights approach to information during crisis" (Signal Program on Human Security and Technology, Harvard Humanitarian Initiative, https://hhi.harvard.edu/sites/default/files/publications/signalcode_final.pdf. 2. Harvard University Privacy Tools and Privacy Insights Project, OpenDP (http://opendp.io).

3. Y. de Montjoye et al., Sci. Data 5, 180286 (2018).

4. P. Maas et al., "Facebook disaster maps: Aggregate in-sights for crisis response \& recovery," Facebook (2019); https://research.fb.com/wp-content/ uploads/2019/04/iscram19_camera_ready.pdf.

5. California Consumer Privacy Act (2020); https://oag.ca.gov/privacy/ccpa.

6. B. Puckett, S. J. McMenemy, "Maintaining employees' privacy during a public health crisis," National Law Review (2020); www.natlawreview.com/article/ maintaining-employees-privacy-during-public-health-crisis.

7. "Statement of the EDPB Chair on the processing of personal data in the context of the COVID-19 outbreak" (2020); https://edpb. europa.eu/sites/edpb/files/files/news/edpb_covid-19_20200316_press_ statement_en.pdf.

8. S. Lai et al., "Assessing spread risk of Wuhan novel coronavirus within and beyond China," medRxiv 10.1101/2020.02.04.20020479 (2020)

9. E. Pollina, D. Busvine, "European mobile operators share data for coronavirus fight," Reuters (2020).

Published online 23 March 2020

10.1126/science.abb8021 